\title{
Eine internationale Landkarte der Personengesellschaften
}

\author{
(einschließlich juristische Personen und Gesamthand)
}

Prof. Dr. Christine Windbichler, LL.M. (Berkeley), Berlin*

Inhaltsverzeichnis

ZGR 2014, 110-138

I. Vorbemerkung und thematischer Überblick . . . . . . . . . . . . . . . . . . . 111

1. Zum Rechtsvergleich . . . . . . . . . . . . . . . . . 111

2. Ausgesparte Bereiche . . . . . . . . . . . . . . . . . . . . 113

3. Vergleichselemente . . . . . . . . . . . . . . . . . 115

II. Verwendungsmöglichkeiten für Personengesellschaften allgemein . . . . . . . . 116

1. Beliebiger Zweck . . . . . . . . . . . . . . . . . . . . . . 116

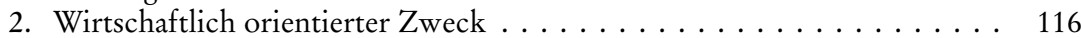

III. Einordnungen und Abgrenzungen . . . . . . . . . . . . . . . . . . 118

1. Vertrag und Schuldrechtsgesellschaft . . . . . . . . . . . . . . . 118

2. Rechtliche Verselbständigung und ,juristische Person“ . . . . . . . . . . 121

3. Personengesellschaft, Körperschaft und Kapitalgesellschaft: fließende Übergänge . . . . . . . . . . . . . . . . . . . . . . . . 125

IV. Bedeutung der Registrierung . . . . . . . . . . . . . . . . . . . . . . . . . . . 127

1. Erwerb der Rechtsfähigkeit . . . . . . . . . . . . . . . . . . 128

2. Vorgesellschaft . . . . . . . . . . . . . . . . . . . . . . 129

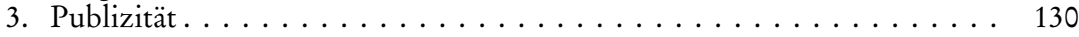

V. Vertretung und das ultra-vires-Problem . . . . . . . . . . . . . . 130

VI. Haftungsverhältnisse . . . . . . . . . . . . . . . . . . 132

VII. Sonderformen für bestimmte Zwecke . . . . . . . . . . . . . . . . . . . 134

VIII. Zusammenfassung: Legende für die Landkarte . . . . . . . . . . . . . . . . 135

Personengesellschaften sind weltweit verbreitet. Bei näherem Hinsehen, hier auf Frankreich Großbritannien und die USA, zeigt sich, dass wesentliche Elemente stark differieren. Es gibt Personengesellschaften obne rechtliche Verselbständigung; eine Trennung des der Gesellschaft gewidmeten Vermögens vom Privatvermögen der Gesellschafter kann dann aber gleichwohl durch die (sachenrechtlichen) Formen gemeinsamer Zuständigkeit erfolgen (Gesamthand, Gemeinschaft, indivision, joint tenancy). Die Einordnung als „juristische Person"

* Prof. Dr. Windiichler ist Inhaberin des Lehrstuhls für Handels- und Wirtschaftsrecht, Arbeitsrecht und Rechtsvergleichung an der Humboldt-Universität zu Berlin. Für wertvolle Materialsammlungen danke ich Frau Dr. Xiao Chen, den Herren Christoph Klaiber, Germain Tchoumbou Bocheng, Ass. jur. Florian Schuhmacher, Dr. Marvin Vesper-Gräske, Matthias Voigt und Ass. jur. Richard Wichmann. Für alle Fehler bin ich allein verantwortlich. 
oder legal entity folgt dem jeweiligen Verständnis dieser Formen. Rechtsfähigkeit und Vertretungsmacht entsprechen sowobl in Frankreich als auch im amerikanischen Recht grundsätzlich dem vertraglichen Unternehmensgegenstand; Anscheinsvollmacht und Vertrauensschutz dienen der Sicherheit des Rechtsverkehrs. Die unbeschränkte und unbeschränkbare Vertretungsmacht ist eine deutsche Besonderheit. Die Registrierung ist teils konstitutiv, teils deklaratorisch, teils zwingend, teils freiwillig. Publizitätsfolgen variieren mit dem jeweiligen Zivil- und Handelsrecht. Der Personenbezug manifestiert sich in einer stärkeren Bindung der Mitgliedschaft als bei juristischen Personen im deutschen Sinne. Die Binnenorganisation zeigt, aus deutscher Sicht, körperschaftliche Züge, etwa beim (Fremd-)Geschäftsführer in Frankreich und dem Mebrheitsprinzip bei der gewöhnlichen Geschäftsführung. Die persönliche Haftung ist durchweg subsidiär, $\int 128$ HGB ist die Ausnabme. Die Vielzabl von Sonderformen einschließlich kapitalmarktgängiger Varianten erklärt sich meist aus dem Stenerrecht. Die Weiterentwicklung des Personengesellschaftsrechts sollte davon ausgehen, welche Elemente für welche Zwecke tanglich sind. Für die formlose, schlichte Vertragsgesellschaft besteht Bedarf, ebenso für den rechtlich verselbständigten Unternehmensträger.

Partnerships are a common form of business association. A closer look however, here to France, the UK and the U.S., reveals substantial differences. Partnerships may be considered an aggregate of their members that does not amount to a legal entity. Nevertheless, funds dedicated to the business can be separated from the partners' patrimony by means of joint tenancy in property law. The concepts of legal entity or legal personality vary considerably. In France as well as in the UK and in the U.S., the powers of a legal entity encompass everything necessary or useful to pursue the (limited) business purpose. Limits in actual authority of persons acting on behalf of the legal entity are subject to agency law including apparent and implied authority. The German concept of mandatory unlimited authority is the exception. Registration constitutes the legal entity, is declaratory, voluntary, or mandatory, as the case may be. Legal consequences as to third party reliance vary with general commercial or civil law. Partnerships are more closely tied to their partners than corporations to their shareholders. The internal organization of the partnership looks more corporate in France, the U.S. and UK than in Germany; examples are majority rule for day-today business decisions and, in France, non-partner officers. Personal liability of partners is usually subordinated; $\int 128$ HGB is a German specialty. The huge variety of partnership forms, including partnerships with listed units, follows tax law incentives. The guiding question for the further development of partnership law is which elements are useful for which purposes. There is demand for a simple, contractual form of partnership as well as for the entity variation as owner of a firm.

\section{Vorbemerkung und thematischer Überblick}

\section{Zum Rechtsvergleich}

Der Titel ist ein wenig merkwürdig. Zum einen ist ja wohl selbstverständlich, dass eine solche Landkarte viele weiße Flecken enthalten muss. Denn der Rechtsvergleicher mahnt sich und andere, mit Originalquellen zu arbeiten, was sofort das Sprachproblem auf den Plan ruft. Eine Weltkarte werde ich daher nicht bieten können und wollen. Englisch und Französisch sind einigermaßen geläufige Sprachen. Nota bene: Englisch ist in den Rechtswissen- 
schaften nicht lingua franca. ${ }^{1}$ Dass ich die österreichischen Personengesellschaften im Folgenden nicht besonders vertiefe, ist kein Sprachproblem, sondern mein tiefer Respekt vor der höheren Kompetenz des Kollegen Schauer. ${ }^{2}$

Gerne verstehe ich „Landkarte“ aber auch als Metapher im Sinne einer Kartierung der Fragen, mit denen wir es bei Personengesellschaften eigentlich zu tun haben. Das rechtfertigt insbesondere der eigenartige Klammerzusatz des Titels. In manchen Ländern sind Personengesellschaften juristische Personen, und die Vermögenszuordnung qua Gesamthand ist auch nicht selbstverständlich. Ferner dienen Kapitalgesellschaften als Referenzpunkt zur Verdeutlichung von Unterschieden und Charakteristika. Das Verständnis, was eine juristische Person ist, ist keineswegs ubiquitär-welteinheitlich. Immerhin: Mit einigem Mut lässt sich behaupten, dass es Personen- und Kapitalgesellschaften so ziemlich überall gibt.

Beim Rechtsvergleich muss man sich stets der Frage stellen, warum man sich der Mühe überhaupt unterzieht. Das allgemeinbildende Interesse daran, wie es in anderen Rechtsordnungen zugeht, resultiert in mehr oder minder erhellenden Länderberichten. Das genügt heutzutage nicht mehr. Darüber, was Rechtsvergleichung ist, sein kann oder sein soll, Methode oder eigenständiger Wissenschaftszweig, deskriptiv, normativ etc., gibt es eine lebhafte Diskussion, ${ }^{3}$ die ich hier nicht aufgreifen will. Pragmatisch verfolge ich folgenden Ansatz. Der Vergleich ist nützlich als Selbstvergewisserung über den Stand der eigenen, nationalen Rechtsentwicklung. Nehmen wir einen Maulwurfshügel als Matterhorn wahr? Haben wir wichtige Fragestellungen entdeckt, guten Antworten zugeführt oder verschlafen? In der grenzüberschreitenden Rechtspraxis sind wir zum Vergleich gezwungen. Exemplarisch nenne ich die autonome Auslegung von Art. 1 Abs. $2 \mathrm{f}$ und g Rom I-VO (Abgrenzung Schuldrecht - Gesellschaftsrecht, keine Rechtswahlfreiheit im Gesellschaftsrecht und Recht der organschaftlichen Vertretung) und ganz allgemein Qualifizierungsfragen im Internationalen Privatrecht. ${ }^{4}$ Außerdem haben wir Entscheidungen zu treffen bei der Rechtsformwahl, die ausländische Rechtsformen einschließt, und anderen Gestaltungsaufgaben. Deshalb frage ich da-

1 Curran, in: Reimann/Zimmermann, The Oxford Handbook of Comparative Law, 2006, S. $675 \mathrm{ff}$.

2 Schauer, in diesem Heft, S. $143 \mathrm{ff}$; zur österreichischen Rechtsentwicklung auch K. Sснмidt, GES 2012, 1; zum schweizerischen Recht Jung, ZVglRWiss 112 (2013), 208 ff; die dort geschilderten „Sonderwege“ mögen rechtfertigen, dass im Folgenden das schweizerische Recht ausgespart bleibt. Zur Kommanditgesellschaft vertieft RöDER, 78 RabelsZ 109 (2014).

3 Vgl. Michaels, in: Reimann/Zimmermann, The Oxford Handbook of Comparative Law, 2006, S. 339 ff; Reimann, 50 Am.J.Comp.L. 671 (2002); WindBichler, FS 200 Jahre Juristische Fakultät der Humboldt-Universität, 2010, S. 1079, 1095 f; ZwEIGERT/KöTZ, Einführung in die Rechtsvergleichung, 3. Aufl., 1996, S. 1-47.

4 Dazu W.H. Roth, in diesem Heft, S. $168 \mathrm{ff}$. 
nach, wozu Personengesellschaften eingesetzt werden, welche Funktionen und Eigenschaften sie haben können, und welche Probleme und Lösungen sich daraus ergeben. Auch das könnte eine Art „Landkarte“ ergeben.

Geht man mit dem Rechtsvergleichern wohlbekannten bome-country bias zunächst von dem geläufigen Grundmuster der Personengesellschaft aus, fände man in Deutschland formfreie vertragliche Grundlage, gemeinsamer Zweck, Abhängigkeit von den jeweiligen (mindestens zwei) Gesellschaftern (d. h. keine freie Übertragbarkeit der Anteile), Selbstorganschaft, Einstimmigkeitsprinzip, persönliche Haftung, weitgehende Gestaltungsfreiheit im Innenverhältnis, grundsätzliche Eignung zur Verselbständigung ( $\$ 124 \mathrm{HGB})$, gesamthänderisch gebundenes Gesellschaftsvermögen und vielleicht Anderes mehr. Dem kann man die Kapitalgesellschaft gegenüber stellen, die juristische Person ist, bestimmte Organe erfordert, Fremdorganschaft erlaubt, dem Mehrheitsprinzip nach Beteiligungshöhe unterliegt, keine Haftung der Gesellschafter vorsieht, kurz: körperschaftlich verfasst ist. Im Folgenden soll die jeweils typische Grundform zugrunde gelegt werden, allerdings mit Blick darauf, welche Regeln zwingend und welche dispositiv sind. Letzteres ist entscheidend für die Flexibilität des Gebildes. Auf gesetzlich geregelte Spezial- und Hybridformen ist nur einzugehen, soweit sie Grundfragen und Regel-/ Ausnahmeverhältnisse beleuchten helfen.

\section{Ausgesparte Bereiche}

\section{a) Die übergestülpte Personengesellschaft}

Nicht zentral sollen hier die „übergestülpten“ Gesellschaften sein, die eher Grenzfälle sind. Damit sind gesellschaftsrechtliche Lösungen gemeint, die eher pragmatisch herangezogen werden, wenn andere Ansätze unzureichend erscheinen. Dazu rechne ich z. B. die Gesellschaft unter Ehegatten oder Lebensgefährten, die ex post konstruiert wird; treibende Kraft ist ein unbefriedigendes Ergebnis des (fehlenden) Familienrechts, die Gesellschaft wird dann als Vehikel zu einem als gerechter empfundenen Ausgleich verwendet. Die vertragliche Grundlage wurde zu diesem Zweck gelegentlich minimiert. ${ }^{5}$

Im Kartellrecht werden Umsätze innerhalb eines Konzerns zur Sanktionsfindung zusammengerechnet und Muttergesellschaften für Töchter in die Haftung genommen, ${ }^{6}$ was im Gesellschaftsrecht auf wenig Gegenliebe stößt. Hier wurde in der Literatur die Zusammenfassung zu einer wirtschaftlichen

5 Vgl. BGH NJW 1999, 2962; zurückhaltender BGH NJW 2012, 33 74, jeweils m. w. N.

6 EuGH, 10. 9. 2009, Rs. C-97/08 P (Akzo Nobel), Slg. 2009 I-08237, Rdn. 42 ff m. w. N.; zur entsprechenden Handhabung nach $\$ 81$ Abs. 4 Satz 3 GWB BGH NJW 2013, 1972, 1975. 
Einheit und Zurechnung als Deutung mit einer GbR deutschen Rechts unter den Konzernunternehmen unterlegt, was zur solidarischen Haftung der Mitglieder führe. ${ }^{7}$ Die Gründung erfolge schlüssig durch gemeinsame Zweckverfolgung; an anderer Stelle heißt es allerdings: die wirtschaftliche Einheit entstehe durch das gemeinsame Auftreten am Markt. Die GbR sei dann die wirtschaftliche Einheit, der der Kartellverstoß anzulasten sei; der Rest ist Haftung. Unabhängig davon, ob deutsches Personengesellschaftsrecht als Deutungsmuster für die Problematik taugt, wird hier der konkludente Gesellschaftsvertrag mit einem Auftreten nach außen begründet, was allenfalls zu einer Scheingesellschaft führt, die allerdings für Bußgelder und Steuern irrelevant ist, da der Staat keinen Verkehrsschutz genießt. Über Einzelheiten lässt sich weiter trefflich streiten; hier will ich nur festhalten, dass ich das Konstrukt in die Kategorie „übergestülpt“ einordne.

Bei der Gläubigerversammlung im Insolvenzverfahren findet man gesellschaftsrechtliche Formen, z. B. den Mehrheitsbeschluss, $\mathbb{S} 5$ 57, 72, 244 InsO, als Regelungsinstrument. Gleichlaufende Interessen (wenn sie denn wirklich gleich laufen) sind aber noch kein „gemeinsamer Zweck“. Deshalb wäre eine gesellschaftsrechtliche Deutung übergestülpt. ${ }^{8} \mathrm{Im}$ Arbeitsrecht taucht das Problem in Form der Führungsvereinbarung beim sog. Gemeinschaftsbetrieb auf, $\mathbb{1} 1$ Abs. 2 BetrVG. ${ }^{9}$

Rechtsvergleichend ist eher die Frage interessant, ob und in welchem Ausmaß andere Rechtsordnungen sich des „Überstülpens“ bedienen und welche Schlüsse daraus gezogen werden können. Das „Überstülpen“ ist womöglich ein Produkt besonders großer Flexibilität, insbesondere der Formfreiheit und der Alternativen im rechtlichen Umfeld.

\section{b) Stewerrecht und Prozessrecht}

Das Steuerrecht ist eine Spezialmaterie, derer ich nicht mächtig bin. Deshalb will ich mich nicht in Vertiefungen wagen, muss es aber gelegentlich erwähnen, da es sich dabei bekanntlich um die ungebetene Rechtsquelle des Gesellschaftsrechts handelt. Insbesondere die Möglichkeit der sog. steuerlichen Transparenz, d. h. Besteuerung der Gesellschafter und nicht der Gesellschaft selbst, treibt oft genug die Rechtsformwahl und reizt zum Ausreizen der Gestaltungsmöglichkeiten. ${ }^{10} \mathrm{Im}$ Rechtsvergleich zeigt sich, dass „Personengesellschaft" und steuerliche „Transparenz" nicht notwendig Hand in Hand

7 Kersting, Der Konzern 2011, 445, 452 (auch: http://ssrn.com/abstract=1884930).

8 Vgl. Ehricke, Münchener Komm. z. InsO, 3. Aufl., 2013, \$74 Rdn. 7 ff.

9 Vgl. BAGE 95, 15 = NZA 2000, 1350 m. w. N.; Koch, Erfurter Komm. z. ArbR,

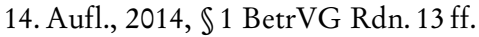

10 Vgl. dazu die Beiträge von Hennrichs, Brandenberg, U. Prinz, Fechner/Bäuml, M. 
gehen, aber doch miteinander zu tun haben. In Frankreich gibt es Wahlrechte, ${ }^{11}$ ebenso in den USA nach dem "check-the-box system “ ${ }^{12}$ Deshalb sei vor einer vorschnellen Annahme des Gleichlaufs von „Personengesellschaft“ und „steuerlicher Transparenz" gewarnt.

Auch das Prozessrecht beherrschen andere besser als ich, daher erwähne ich es nur in Form von Fragestellungen: Wen kann oder muss man ggf. verklagen? Wie findet man heraus, wen man sinnvollerweise verklagen sollte: Gesellschafter und/oder Gesellschaft, auffindbar über ein Register? Was geschieht bei einem Gesellschafterwechsel, falls der überhaupt zulässig ist, während eines Aktiv- oder Passivprozesses? All das hängt natürlich mit den Eigenheiten der jeweiligen nationalen Prozessrechte zusammen und ist ein Thema für sich.

\section{Vergleichselemente}

Folgende Elemente will ich einer näheren Betrachtung unterziehen. Zunächst ist festzustellen, zu welchen Zwecken Personengesellschaften von Gesetzes wegen zur Verfügung stehen. Im deutschen Recht kann ein beliebiger gemeinsamer Zweck in Form einer Personengesellschaft verfolgt werden. Das ist nicht überall so. Damit hängt auch die Frage zusammen, wie sich eine Gesellschaft von einem gewöhnlichen Vertrag unterscheidet.

Natürlich ist der Frage nach der rechtlichen Verselbständigung nachzugehen, was eine gewisse Vorsicht erfordert, denn unter den Begriffen „Rechtsfähigkeit", „juristische Person“, „personne morale, personnalité juridique“, „legal person, legal entity" verbergen sich durchaus verschiedene Verständnisse. Registrierungserfordernisse haben unterschiedliche Voraussetzungen und Folgen. Auch die Unterscheidung der Personen- von den Kapitalgesellschaften zeigt fließende Übergänge, denen einige Aufmerksamkeit gebührt. Ferner werden die Fungibilität der Anteile und die Binnenorganisation, die mehr vertraglich, mehr personalistisch oder mehr körperschaftlich sein kann, betrachtet. Im Außenverhältnis interessieren Vermögensträgerschaft, Vertretung und Haftung. Die Landkarte ist hier nicht weniger bunt.

Schmitt und Richter/Welling zum 35. Berliner Steuergespräch, FR 2010, $721 \mathrm{ff}$; rechtsvergleichend zu Personengesellschaften Hey/Bauersfeld, IStR 2005, 649.

11 Dazu näher Kussmaul/Schäfer, IStR 2000, 161.

12 HaYes, 54 Wash.\&Lee L.Rev 1147 (1997); aktuelles Formular: http://www.irs.gov/pub/ irs-pdf/f8832.pdf. 
II. Verwendungsmöglichkeiten für Personengesellschaften allgemein

\section{Beliebiger Zweck}

Aus dem deutschen Recht sind wir gewöhnt, dass die Personengesellschaft in ihrer Grundform, der Gesellschaft bürgerlichen Rechts, für jeden beliebigen gemeinsamen Zweck verwendet werden kann, mit Ausnahme des Handelsgewerbes, das automatisch in die OHG führt, aber auch die KG eröffnet; die PartG (und PartGmbB) steht ausschließlich für freie Berufe zur Verfügung. Die OHG, mit dem reichen Erfahrungsschatz des Handelsrechts im Hintergrund, ist gleichwohl die klarere, ausgereiftere Form. Trotz $\$ 105$ Abs. 3 HGB ist der Griff ins Handelsrecht zur Lösung von Fragen der GbR beliebt; auch der Gesetzgeber folgte dieser Umkehrung der Verhältnisse in $\$ 736$ Abs. 2 BGB. Dass die GbR Grundform und Rückfallposition ist, steht dennoch außer Zweifel. Das gilt für die nicht eingetragene kleingewerbliche Gesellschaft ebenso wie für die (noch) nicht eingetragene Partnerschaft. Aber auch die OHG ist zunächst Personengesellschaft allgemeiner Art, für die das HGB dann Sonderregeln vorsieht - oder vorsah, wenn man von der zwischenzeitlichen Rechtsfortbildung absieht. Das erklärt, warum die heftige Debatte um des „Wesen“ der Gesamthand am Beispiel der GbR geführt wurde, denn für die OHG waren die wichtigsten Fragen spezialgesetzlich geregelt. Das gilt insbesondere für $\$ 124 \mathrm{HGB}$, eine Vorschrift, die nach dem Gesetzestext des alten $\$ 105$ Abs. 2 und jetzigen $\$ 105$ Abs. 3 HGB konstitutive Kraft hat. ${ }^{13}$

\section{Wirtschaftlich orientierter Zweck}

Das französische Recht kennt die société civile (SC) und die société en nom collectif (SNC), letztere vergleichbar der OHG und auf den Betrieb eines Handelsgewerbes gerichtet. Die einfachste Variante der Personengesellschaft ist die société en participation. Selbst diese wie auch die société civile verlangen einen gemeinsamen Zweck in dem Sinne, dass die Vorteile der gemeinsamen Aktivität oder Ersparnisse geteilt werden. Darin liegt eine wirtschaftliche Komponente, die aber nicht Unternehmensträgerschaft bedeuten muss. ${ }^{14}$ Als Anwendungsbeispiel für die société en participation wird die Lottotippgemeinschaft genannt. ${ }^{15}$ Der gemeinsame Zweck kann genossenschaftsähnlich

13 A. HuEck, Das Recht der offenen Handelsgesellschaften, 4. Aufl., 1971, S. 216 ff, 271 ff; zur Entwicklung der gegenteiligen (herrschenden) Auffassung K. SснміDT, AcP 209 (2009), 181, 184 ff; vgl. auch Wiedemann, Gesellschaftsrecht, Band 2, 2004, \$8 III 1.

14 Wegen ihrer praktischen Bedeutung behandeln RIPERT/RoвLOT/GERMAIN/VOGEL, Les sociétés commerciales, in: Ripert/Roblot, Traité de droit Commercial, Tome 1 Vol. 2, 18. Aufl., 2002, Rdn. 1170 die société en participation unter den Handelsgesellschaften.

15 Nach Cozian/Viandier/Deboissy, Droit des sociétés, 21. Aufl., 2008, Rdn. 1215 Fn. 4: Cour de cass. 1e civ., 14.1.2003: JCP E 2003, 763. 
sein; eine besondere Ausprägung ist die Spezialform des groupement d'intérèt économique (Vorbild für die EWIV). Die handelsrechtlichen Gesellschaftsformen (SA, SARL, SCpA etc.), mit Ausnahme der SNC, stehen für alle unternehmerischen Tätigkeiten offen, auch für freie Berufe (professions libérales), für die dann sondergesetzliche Modifikationen eingreifen. ${ }^{16}$ Die société civile kommt für freiberufliche, künstlerische und landwirtschaftliche Tätigkeiten in Betracht; sie ist Auffangregel. Freiberufler, Künstler und Landwirte sind keine „commerçants“, was aber an der wirtschaftlichen Ausrichtung im weiten Sinne nichts ändert. Für nichtwirtschaftliche $Z$ wecke gibt es die association. ${ }^{17}$

Im englischen Recht ist die general partnership die einfachste Form, gemeinsam eine geschäftliche Tätigkeit zu betreiben (carry on a business). ${ }^{18}$ Unter „business“ ist nicht notwendig ein Gewerbe in unserem Sinn zu verstehen; es genügt ein limitiertes Projekt (vgl. ARGE) mit Gewinnerzielungsabsicht. ${ }^{19}$ Eine Unterscheidung zwischen Handelsgesellschaften und Gesellschaften bürgerlichen Rechts (vgl. société civile) gibt es nicht. Auch im amerikanischen Recht (nach dem Revised Uniform Partnership Act - RUPA, mit Varianten in den einzelnen Staaten) ist es ähnlich: Die Definition der „partnership“ enthält das Element der gemeinsamen Kontrolle über ein „business“, das auch aus einem einzelnen Projekt bestehen kann (z. B. Bebauung eines Grundstücks und Verwertung der Immobilie). Eine „Gesellschaft bürgerlichen Rechts“ gibt es nicht.

Das englische und das amerikanische Recht ${ }^{20}$ kennen keine klare Unterscheidung zwischen bürgerlichem und Handelsrecht. Hier ist das Interessante an der partnership etwas Anderes: Im Umfeld eines im Vergleich zu unserem sperrigeren Vertragsrecht (Stichworte: consideration, deed, parole evidence rule) können partnerships als Konsensualverträge auch konkludent und ohne Rücksicht auf die Bezeichnung ${ }^{21}$ durch die Parteien gebildet werden. Der

16 Cozian/Viandier/Deboissy, aaO (Fn. 15), Rdn. 1128, $1262 \mathrm{ff}$.

17 Dazu Cozian/Viandier/Deboissy, aaO (Fn. 15), Rdn. 62 ff; C. Frey, Die Vertretung verselbständigter Rechtsträger in europäischen Ländern Teil VI Frankreich, 2003, S. $102 \mathrm{ff}$.

18 Sec. 1 (1) Partnership Act 1890: "Partnership is the relation which subsists between persons carrying on a business in common with a view of profit."

19 Vgl. zum englischen Recht: LindLey \& BANKs on Partnership, 18. Aufl., 2002, Rdn. 2-02.

20 Ich erlaube mir hier, vom amerikanischen Recht zu sprechen, weil die ganz große Mehrzahl der Einzelstaaten, wenn auch mit Unterschieden im Detail, die Uniform Acts, Uniform Partnership Act (UPA) von 1914, gefolgt 1996 vom Reformed Partnership Act (RUPA) [http://www.uniformlaws.org/shared/docs/partnership/upa_final_97.pdf], übernommen haben.

21 Vgl. $\$ 7$ Abs. 4 UPA, $\$ 202$ a RUPA; Martin v Peyton, 246 N.Y. 213, 158 N.E. 77 (1927); dazu W.A. KLEIN, http://papers.ssrn.com/sol3/papers.cfm?abstract_id=1088494 (2008). 
Aufhänger ist die gemeinschaftliche wirtschaftliche Betätigung. Voraussetzung ist, dass tatsächlich eine geschäftliche Aktivität betrieben wird. ${ }^{22}$

Ob Personengesellschaften mit einer Vielzahl von Gesellschaftern zu Anlagezwecken zulässig oder gar vorgesehen sind, variiert stark. In Frankreich gibt es spezialgesetzliche Regelungen für ebendiese Form im Immobilienbereich, ${ }^{23}$ in den USA im Bereich der Energiewirtschaft. Treibende Kraft ist hier oft der Zugang zur steuerlichen Transparenz. ${ }^{24}$

Als Zwischenergebnis halte ich deshalb fest: Die Landkarte soll sich auf Personengesellschaften mit einer wirtschaftlichen Betätigung (nicht notwendig Gewerbe oder gar Handelsgewerbe) beschränken. Die Abrede, gemeinsam einen jungen Künstler zu fördern, ${ }^{25}$ wird hier also nicht verzeichnet. Das bedeutet nicht, dass eine solche Aktivität in anderen Ländern nicht ginge, sondern nur, dass es sich nicht um eine Personengesellschaft handelt. Dem entspricht auch Art. 54 AEUV, der die Niederlassungsfreiheit den Gesellschaften mit Erwerbszweck garantiert, wobei der Erwerbszweck weit zu verstehen ist, also Genossenschaften einschließt und kein Gewerbe erfordert. Der deutschen Rechtsentwicklung ist das nicht ganz fremd; z. B. hat Jung die Deutung von (unternehmenstragenden) Gesellschaften vom „Unternehmergesellschafter" her unternommen. ${ }^{26}$ Der Gewerbebegriff steht seit längerem in der Kritik; ${ }^{27}$ Instanzgerichte haben auch schon versucht, die ARGE als OHG zu deuten. ${ }^{28}$ Freiberufler sind jedenfalls Unternehmer.

\section{Einordnungen und Abgrenzungen}

\section{Vertrag und Schuldrechtsgesellschaft}

Die Gesellschaft bürgerlichen Rechts steht im Besonderen Schuldrecht, sie kann also „reines“ Schuldverhältnis sein. Darauf ist sie aber offenkundig nicht beschränkt. Die Stille Gesellschaft steht im HGB, ist aber keine Handelsgesellschaft. Sie wird jedenfalls in ihrer gesetzlichen Form, also nicht der

22 Zum englischen Recht: Lindley \& Banks, aaO (Fn. 19), 2-02 f, 2-13.

23 Cozian/Viandier/Deboissy, aaO (Fn.15), Rdn. $1210 \mathrm{~d}$ : société civile de placement immobilier.

24 Cozian/Viandier/Deboissy, aaO (Fn.15), Rdn.1170; zum Steuerrecht oben Fn. 10-12.

25 So das Beispiel bei A. Hueck, Gesellschaftsrecht, 1. Aufl., 1948, S. 22.

26 Jung, Der Unternehmergesellschafter als personaler Kern der rechtsfähigen Gesellschaft, 2002.

27 K. Schmidt, DB 1994, 515 ff; Ders., Münchener Komm. z. HGB, 3. Aufl., 2010, $\$ 1$ Rdn. 4 m.w. N.

28 KG BauR 2001, 1790; OLG Frankfurt, BeckRS 2005, 00674; Mantler, Münchener Handbuch des Gesellschaftsrechts, Band 1, 3. Aufl., 2009, $\$ 26 \mathrm{Rdn} .20 \mathrm{ff}$. 
typisch-atypischen oder atypisch-atypischen stillen Gesellschaft, nicht als Gesellschaft i. S.d. Art. 1 Abs. 2 f Rom I-VO angesehen, sondern als Vertrag. ${ }^{29}$ Nach Art. 54 AEUV ist „Gesellschaft“ alles, was erwerbstätig und nicht natürliche Person ist, ${ }^{30}$ also ebenfalls nicht die stille Gesellschaft.

Charakteristisch für solche reinen Vertragsgesellschaften ist, dass sie Dritten gegenüber kein eigenes Gesellschaftsvermögen haben und nicht nach außen hervortreten. Im französischen Recht ist die einfachste Variante der Personengesellschaft, die société en participation (SEP), eine solche Vertragsgesellschaft. Außengesellschaft kann sie nach - bestrittener - h. M. nicht sein. ${ }^{31}$ Jedenfalls bedarf es eines Vertrages zu einem gemeinsamen Zweck (affectio societatis) und Beiträgen der Gesellschafter. Sie wird nicht im Régistre du Commerce et des Sociétés (RCS) eingetragen; Handelsregister mag ich dazu nicht sagen, denn die „sociétés" sind hier auch die sociétés civiles. Ein Standardlehrbuch bringt das so auf den Punkt: Die société en participation verhält sich zu den eingetragenen Gesellschaften wie das Konkubinat zur Ehe. ${ }^{32}$ Anwendungsfälle für diese Gesellschaftsform sind neben der Lottotippgemeinschaft Unternehmenskooperationen, die Finanzierung eines riskanten Vorhabens oder Emissionskonsortien und andere Bankenpools (genannt syndicat financier)..$^{33}$ Ferner wird genannt die „stabilisation d'une indivision“. Im Wörterbuch wird „indivision“ mit „Gesamthandsgemeinschaft" übersetzt, was aber nicht wörtlich genommen werden darf; es handelt sich um eine Art gemeinschaftlichen Eigentums, besser als Rechtsgemeinschaft bezeichnet. ${ }^{34}$ Ausgangspunkt war die noch ungeteilte Erbengemeinschaft, eine grundsätzlich jederzeit beendbare, eher bruchteilsartige gemeinschaftliche Berechtigung, C.civ. art. $815 \mathrm{ff}$, nunmehr generell geregelt in Artt. 1873-1 ff C.civ. ${ }^{35}$ In diesen Fällen gibt es also ein "Gesellschaftsvermögen“ (substitut de patrimoine opposable aux tiers), ${ }^{36}$ das für das Innenverhältnis maßgebend ist und dessen Teilung vor

29 Vgl. BGH NJW 2004, 3706.

30 Forsthoff, in: Grabitz/Hilf/Nettesheim, Recht der Europäischen Union, 2013, Art. 54 AEUV Rdn. 4; Michalski/Funke, GmbHG, 2. Aufl., 2010, $\$ 4$ a Rdn. 72.

31 Die Offenlegung des Gesellschaftsverhältnisses ist freilich möglich; vgl. Cozıan/VianDier/Deboissy, aaO (Fn. 15), Rdn. 1224 f; C. Frey, aaO (Fn. 17), S. 153 f; H. Nitschke, Das Recht der Personengesellschaften in Deutschland und Frankreich, 2001, S. 138 ff; handeln die Gesellschafter Dritten gegenüber als solche, folgt daraus persönliche Haftung, C.civ. art. 1872-1. - Die SEP kann aber für die Besteuerung der Gesellschaft selbst votieren.

32 Cozian/Viandier/Deboissy, aaO (Fn. 15), Rdn. 1213.

33 Cozian/Viandier/Deboissy, aaO (Fn. 15), Rdn. $1215 \mathrm{ff}$.

34 Ferid/Sonnenberger, Das französische Zivilrecht Bd.2, 2. Aufl., 1986, 2L26; zu den sprachlichen Klippen H. NitschKe, aaO (Fn. 31), S. $161 \mathrm{f}$.

35 Dazu H. Nitschke, aaO (Fn. 31), S. 61 ff, zur Geschichte S. $106 \mathrm{ff}$.

36 Zur Überlagerung von Gesellschaft und indivision Ferid/SONNENBerger, aaO (Fn. 34), 2L501; Nitschke, aaO (Fn. 31), S. 142 ff; Cozian/Viandier/Deboissy, aaO (Fn. 15), Rdn. 1222, 1232. 
Auflösung der Gesellschaft nicht verlangt werden kann. Im Außenverhältnis bleibt es beim Recht der indivision. Als Beispiele werden eine gemeinsame Raffinerie mehrerer Mineralölunternehmen oder Emissionskonsortien genannt. Da die SEP nicht rechtlich verselbständigt ist, unterfällt sie auch nicht dem französischen Unternehmensstrafrecht. ${ }^{37}$

Im englischen Recht (England und Wales) tut man sich schwer, reine Innengesellschaften zu finden. Der Normalfall ist die Außengesellschaft. Die vertragliche Grundlage kann formlos, auch konkludent sein. ${ }^{38}$ Gleichwohl wird die general partnership nicht als reine Vertragsbeziehung angesehen, sondern zugleich als persönliche und geschäftliche Beziehung. ${ }^{39}$ Das betrifft aber wiederum nur das Innenverhältnis. Im Außenverhältnis sind die partner Träger von Rechten und Pflichten, was mit aggregate approach bezeichnet wird im Gegensatz zum entity approach (rechtliche Verselbständigung). ${ }^{40} \mathrm{Da}$ fühlt man sich stark an die deutsche GbR alten Zuschnitts erinnert. Der Innengesellschaft am nächsten kommt man wohl mit der partnership mit einem sog. "dormant partner“. ${ }^{41}$

Entsprechendes galt für die amerikanische partnership nach dem Uniform Partnership Act (UPA) von 1914. Amerikanische Lehrbücher zu „business associations" fangen normalerweise mit ,agency“ an, "agency and partnership“ ist eine geläufige Paarung im Angebot der Darstellungen; association ist ein neutraler Begriff, der tatsächliches Zusammenwirken, Vertrag und juristische Person umfasst. Das hat nicht nur mit der ökonomischen Betrachtung von Mehrpersonenverhältnissen im wirtschaftlichen Zusammenhang $\mathrm{zu}$ tun, ${ }^{42}$ sondern auch damit, dass der handelnde partner beim aggregate approach die anderen partners, sonst die partnership vertritt. Der Vergleich mit älteren

37 Cozian/Viandier/Deboissy, aaO (Fn. 15), Rdn. 249, 1213 a.E.

38 The Law Commission (LAW COM No.283) and The Scottish Law Commission (SCOT LAW COM No. 192), Gemeinschaftsbericht zum Partnership Law (Nov. 2003, Reference number: LC283), S. 6 (abrufbar unter http://lawcommission.justice.gov.uk/ publications/partnership-law.htm), im Auftrag des Department of Trade and Industry (Wirtschaftsministerium), jetzt: Department of Business, Innovation and Skills (im Folgenden: Partnership Law, Gemeinschaftsbericht 2003); Morse, Partnership Law, 7. Aufl., 2010, Rdn.1.11.

39 Lord Millett, in: Hurst v Bryk [2002], 1 A.C. 185, 194; Partnership Law, Gemeinschaftsbericht 2003, aaO (Fn. 38), S. 6; Lindley \& Banks, aaO (Fn. 19), 2-13; Barron, Company Lawyer 2004, 25(10), 293.

40 Vgl. Partnership Law, Gemeinschaftsbericht 2003, aaO (Fn. 38), S. 6-8, 45 ff (mit ausführlicher Kritik zum aggregate approach).

41 Vgl. Lindley \& Banks, aaO (Fn. 19), 12-08.

42 Jennings/Buxbaum, Corporations, 5. Aufl., 1979, S. 1: The least structured form of association is that of principal and agent: a person acting on behalf of another person; weitergehend M. A. Eisenberg, Corporations and Other Business Organizations, 9. Aufl., 2005, S. 1 f, der selbst das Einzelunternehmen als „business organization“ bezeichnet. 
Gesamthandslehren hinkt aber, denn „agency“ ist nicht identisch mit Stellvertretung, sondern eine vertragliche Beziehung, die zu einer gegenüber dem gewöhnlichen Vertrag verschärften Treuepflicht, einer fiduciary duty, führt. ${ }^{43}$ Die bloße Vertretungsmacht bezeichnet man mit authority. Eine agency-Beziehung ist also eine besondere Vertragsart, die weder Entgeltlichkeit noch Form voraussetzt. Auf die Vertretungsfragen komme ich noch gesondert zurück. Der Reformed Partnership Act (RUPA) von 1997 ging zur rechtlichen Verselbständigung mit etlichen personalistischen Elementen über; dazu unten III.2.

Besonders bei zweigliedrigen Beziehungen ist die Unterscheidung zwischen (Vertrags-)Gesellschaft und gewöhnlichem Vertrag (mit konkretisierender Ausgestaltung) schwierig; die Bezeichnung ist nicht entscheidend. Das betrifft z. B. Finanzierungsvereinbarungen, in denen der Geldgeber Kontroll- und Zustimmungsrechte hat. Folgende Gesichtspunkte lassen sich als Gemeinsamkeiten im Rechtsvergleich feststellen: der gemeinsame Zweck (affectio societatis), Einfluss auf die Geschäftsführung und die Verlustbeteiligung. ${ }^{44}$ Daraus folgt, dass covenants mit Kontroll- und Zustimmungsrechten, die von manchen schon als (Innen-)gesellschaften angesehen werden, keine Gesellschaften sind.

Demnach sind Vertrag und juristische Person keine Gegensätze; auch die Satzung ist Rechtsgeschäft. Die Frage muss eher lauten, ob durch oder auf der Grundlage des Vertrages eine nach außen relevante Organisation geschaffen wird. ${ }^{45}$

\section{Rechtliche Verselbständigung und "juristische Person“}

Vom gewöhnlichen Vertrag zur Gesellschaft mit affectio societatis und Treuepflicht vorangeschritten, stellt sich die Frage nach der rechtlichen Verselbständigung. Ich spreche ganz bewusst erst einmal von rechtlicher Verselbständigung, denn, wie schon erwähnt, sind juristische Person, personne morale, legal person etc. nicht dasselbe. Der Sache nach geht es zunächst darum, ob die Gesellschaft selbst Vermögensträger ist und Rechte und Pflichten haben kann.

43 Eisenberg, aaO (Fn. 42), S. 2; Restatement (Third) of Agency, 2007, $\mathbb{} 1$.

44 In Davis v LofTus, 334 Ill. App. 3 d 761, 778 N.E.2 d 1144) (2002), wurden „partner“ einer Anwalts-partnership nach diesen Kriterien als Angestellte, nicht als partner angesehen. Wenn sich Kontrollrechte des Geldgebers nur auf die Sicherung seines Rückzahlungsinteresses beziehen, handelt es sich um ein (partiarisches) Darlehen, nicht um eine partnership, vgl. Martin v Peyton, aaO (Fn.21); Cozian/Viandier/Deboissy, aaO (Fn. 15), Rdn. 1219; Einflussnahme des Geldgebers auf den Schuldner kann aber zur Haftung als Geschäftsherr (principal) in einem agency-Verhältnis führen, vgl. GAY Jenson Farms Co. v Cargill, Inc., 309 N.W.2 d 285 (Minn. 1981).

$45 \mathrm{Zu}$ dieser Fragestellung vgl. K. SснміDт, ZHR 177 (2013), 712, $720 \mathrm{ff}$. 
Für unternehmenstragende Gesellschaften ist das praktisch unerlässlich. Im deutschen Recht erreichen das $\ 124$ HGB, die Verweisungen darauf (PartGG) und die Rechtsfortbildung zur BGB-Gesellschaft. Letztere ist m.E. nicht abgeschlossen, denn die Voraussetzungen und Folgen im Einzelnen lassen noch einige Fragen offen. ${ }^{46}$ Eine andere Frage ist, ob die Verselbständigung als „juristische Person“ auch Unabhängigkeit vom Gesellschafterbestand bedeutet. ${ }^{47}$

Zum englischen Recht hat die Law Commission 2003 bitter beklagt, dass die general partnership nach dem Partnership Act von 1890 keine von den Gesellschaftern getrennte Einheit ist und nicht Vermögensträger sein kann. ${ }^{48}$ Es kommen als "Gesellschaftsvermögen“ also nur die allgemein anerkannten Formen des gemeinschaftlichen Eigentums in Betracht (tenancy in common, joint tenancy); oder man errichtet einen trust. ${ }^{49} \mathrm{Da}$ die Vorschläge der Commission nicht umgesetzt wurden, ist auch heute noch der Rechtszustand der "aggregate approach" ${ }^{50}$ also wie im UPA oder auch die „common law theory“. Die limited partnership (LP), nach common law unbekannt, wurde durch den Limited Partnership Act von 1907 eingeführt. ${ }^{51}$ Sie ist ebenfalls nicht rechtlich verselbständigt. ${ }^{52}$ Die UK LLP, geschaffen durch den Limited Liability Partnership Act von 2000 (Anwendungsbereich: England, Wales, Schottland und Nordirland), hat Rechtspersönlichkeit und ist stark der $\mathrm{GmbH}$ angenähert, sie wird auch als body corporate bezeichnet. ${ }^{53} \mathrm{Im}$ schottischen Recht ist bereits die general partnership ,a legal person distinct from the partners“; ;4 sie ist Ver-

46 Windbichler, Gesellschaftsrecht, 23. Aufl., 2013, \2 Rdn. 4, 6, \5 Rdn. 6; für eine Grundsatzdiskussion etwa Beuthien, NJW 2005, 715; K. Sснмidt, ZIP 2014, 493, $497 \mathrm{ff}$.

47 So zum deutschen Recht die Unterscheidung zwischen Personengesellschaft und Körperschaft, vgl. Ulmer/Schäfer, Münchener Komm. z. BGB, 6. Aufl., 2013 , \705 Rdn. 308 f; WindiIchler, aaO (Fn. 46), \$2 Rdn. 7; zur Schwierigkeit, Strukturelemente deutscher Gesellschaftsformen zu systematisieren, Hadding, Festgabe Zivilrechtslehrer 1934/1935, 1999, S. 147.

48 Oben Fn. 38, S. 45 ff, 145; prägnante Zusammenfassung im Summary, S. 1-2.

49 Vgl. Sec. 20 und 21 des Partnership Act 1890; kritisch Partnership Law, Gemeinschaftsbericht 2003, aaO (Fn. 38), S. 145 f; Lindley \& Banks, aaO (Fn. 19), 18-02, 18-69.

50 Blackett/Haren, Partnership Law, 4. Aufl., 2011, Rdn. 1.9; Milman, 339 Company Law Newsletter 2013, 4 m. w. N. zur Rspr.

51 Lindley \& Banks, aaO (Fn. 19), 28-01, 28-06.

52 Lindley \& Banks, aaO (Fn. 19), 29-01; Milman, aaO (Fn. 50), m.N. zur Rspr.

53 Sec. 1 (2) LLP Act 2000; vgl. Lord Mackay of Clashfern, Halsbury's Laws of England, Bd.79, 5. Aufl., 2008, Rdn. 234; Milman, aaO (Fn. 50); Blackett/Haren, aaO (Fn. 50), 25.4; zur Entwicklung M. Kilian, NZG 2000, 1008; SCHAPER, ECFR 2013, 75, $94 \mathrm{ff}$.

54 Partnership Act 1890, Sec. 4(2): "In Scotland a firm is a legal person distinct from the partners of whom it is composed,...". 
mögensträger, kann klagen und verklagt werden..$^{55}$ Gleichwohl ist sie in stärkerem Maße vom Mitgliederbestand abhängig als wir es bei einer juristischen Person vermuten würden. ${ }^{56}$

Im amerikanischen Recht folgte die Revision des UPA durch den Revised Uniform Partnership Act von 1997 (RUPA) explizit der entity theory. Historisch interessant mag sein, dass bereits der erste Entwurf zum UPA 1902 auf Betreiben des Kommissionsvorsitzenden der entity theory folgte. Da aber der Vorsitzende vor Abschluss der Arbeiten starb und sein Nachfolger, ein vehementer Anhänger der aggregate theory, die Kommission von seiner Theorie überzeugte, blieben im UPA die Gesellschafter die Rechtsträger. ${ }^{57}$ Dabei machte bereits der UPA vielfache Zugeständnisse und behandelte die partnership wie eine Einheit; umgekehrt enthält der RUPA „aggregate-like results on certain issues". ${ }^{58}$ Melvin Eisenberg fasst das trocken so zusammen: Kein Ergebnis zu einer spezifischen Frage zum partnership law kann „logisch“ oder „notwendig“ aus dem legal-entity status oder dessen Verweigerung „abgeleitet" werden. Die Verfasser des RUPA haben in jeder Einzelfrage rechtspolitische Entscheidungen getroffen; die rechtliche Verselbständigung als Ausgangspunkt vereinfacht Vieles, ist aber nicht abschließend. ${ }^{59}$ Der Übergang zur entity theory verlangt $\mathrm{z}$. B. eine genauere Abgrenzung zum joint venture, das nach common law und UPA sehr partnerschaftsähnlich war, aber auch heute noch eine spezielle vertragliche Verbindung und keine entity ist. ${ }^{60}$ Die

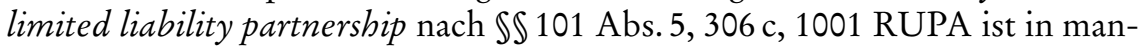
chen Staaten, ähnlich der britischen LLP, nur für Freiberufler verfügbar. ${ }^{61}$

Im französischen Recht sind die société civile und die société en nom collectif personne morale, also ,juristische Personen“. Das steht im Code civil und im Code de commerce. Die Vermögensträgerschaft ist also klar. Bedeutet das aber unabhängige Existenz vom Kreis der Gesellschafter? Die Bestimmung der Identität der Gesellschaft anhand der Gesellschafter spiegelt sich in der Möglichkeit der Veränderung des Gesellschafterkreises und der grundsätzlichen (Un-)Übertragbarkeit der Anteile.

55 Vgl. Partnership Law, Gemeinschaftsbericht 2003, aaO (Fn.38), S. 7; Milman, aaO (Fn. 50).

56 Vgl. Partnership Law, Gemeinschaftsbericht 2003, aaO (Fn. 38), S. 7.

57 M. A. Eisenberg, aaO (Fn. 42), S. 41.

58 M. A. Eisenberg, aaO (Fn. 42), S. 42

59 M. A. Eisenberg, aaO (Fn. 42), S. 42

60 M. A. Eisenberg, aaO (Fn. 42), S. 87 f; O`Kelley/Thompson, Corporations and Other Business Associations, 4. Aufl., 2003, S. 49; anders noch Jennings/Buxbaum, aaO (Fn. 42), S. $31 \mathrm{ff}$ : The joint venture: a variation on the partnership.

61 Z.B. in Kalifornien und New York, M. A. Eisenberg, aaO (Fn. 42), S. 511; Klein/ Ramseyer/Bainbridge, Business Associations, 6. Aufl., 2006, S.299; O'Kelley/ Thompson, aaO (Fn. 60), S. 50 f, 52; zur Entwicklung Henssler, FS Wiedemann, 2002, S. 907, 909 ff; Schaper, aaO (Fn. 53), 75, 86 ff. 
Die englische, nicht rechtlich verselbständigte general partnership wird automatisch aufgelöst durch jedes Ausscheiden eines Gesellschafters, ${ }^{62}$ was als erheblicher Nachteil empfunden wird, denn diese common law-Regel ist nicht einmal dispositiv. ${ }^{63}$ In der limited partnership sind dagegen die Mitgliedschaften der limited partners - mit Zustimmung der general partners - abtretbar. ${ }^{64}$ Nota bene: die limited partnership ist durch Gesetz von 1907 ohne Vorbild im common law in Anlehnung an die französische société en commandite geschaffen worden und hat bis heute kein große Verbreitung gefunden. ${ }^{65}$

Der amerikanische UPA folgte der common law-Regel, die Neufassung 1997 RUPA weicht davon ganz erheblich ab. Eine Reihe von Ereignissen führt zum Ausscheiden eines Partners gegen Abfindung; es gibt sogar ein unbedingtes Austrittsrecht, das bei Missbrauch zu Schadensersatzansprüchen führt, $\$ 601$ RUPA. Bei Auflösungsgründen gibt es Fortsetzungsmöglichkeiten, $\int \mathbb{S} 801$, 802 b RUPA. Da liegt es nahe, die Handelsrechtsreform von 1998 in Erinnerung zu rufen: Der neue $\$ 131$ Abs. 3 HGB ist eine ganz erhebliche Verselbständigung der Gesellschaft vom Bestand ihrer Gesellschafter. Dass für den Fall des Todes eines Gesellschafters keine Fortsetzungsklausel mehr erforderlich ist, ist so naheliegend, dass die darin liegende dogmatische Änderung kaum thematisiert wurde. ${ }^{66}$ In den USA war und in England ist das ein echter Diskussionspunkt.

Das französische Recht dagegen hat bei der société civile und der société en nom collectif, die ja personne morale sind, hinsichtlich der Identität kein Problem mit dem einverständlichen Ausscheiden von Gesellschaftern. Nicht einmal das Ausscheiden des vorletzten Gesellschafters führt zur Auflösung, wenn innerhalb eines Jahres wieder ein weiterer Gesellschafter hinzukommt, C.civ. art. 1844-5. Aber auch die société en participation, die Vertragsgesellschaft, wird durch das Ausscheiden eines Gesellschafters nicht aufgelöst, da C.civ. art. 1871-1 auf das Recht der eingetragenen Personengesellschaften, je nach Gesellschaftszweck civile oder en nom collectif, verweist. Eine Bindung an die Gesellschafter zeigt sich aber darin, dass es grundsätzlich ${ }^{67}$ keine Kün-

62 Partnership Law, Gemeinschaftsbericht 2003, aaO (Fn. 38), S. 7 m.N. zur Rspr.; LindLEY \& BANKs, aaO (Fn. 19), 3-04.

63 Vgl. Partnership Law, Gemeinschaftsbericht 2003, aaO (Fn. 38), S. 7 m.N. zur Rspr.; Summary zum Partnership Law, Gemeinschaftsbericht 2003, S. 2.

64 Sec. 6 (2)(b) Limited Partnerships Act 1907.

65 Vgl. Blackett/Haren, aaO (Fn. 50), 1.5; Lindley \& Banks, aaO (Fn. 19), 28-01 f, 28-09; RÖDER, 78 RabelsZ 109, $116 \mathrm{ff.}$

66 Krit. unter Hinweis auf Streitpotenzial mit Erben K. Sснміdт, aaO (Fn. 27), \131 HGB Rdn. 63; rechtsvergleichend hängt diese Einschätzung davon ab, ob ein Fortsetzungsbeschluss bei aufgelöster Gesellschaft möglich ist und welche erbrechtlichen Vorschriften eingreifen, vgl. Cozian/Viandier/Deboissy, aaO (Fn. 15), Rdn.1156f.

67 Bei der société civile gibt es einen Austritt aus wichtigem Grund, der gerichtliche Autorisierung verlangt, C.civ. art. 1869. 
digung der Mitgliedschaft gibt. Sind die Mitgesellschafter mit dem Ausscheiden nicht einverstanden, bleibt dem Dissidenten nur die Auflösungsklage. Dass die Veräußerung von Gesellschaftsanteilen der Zustimmung aller Gesellschafter bedarf, ist für die société en nom collectif zwingend, C.com. art. L221-13; die société civile eröffnet dagegen Spielräume für die gesellschaftsvertragliche Ausgestaltung, C.civ. art. 1861. Die société civile wird durch den Tod eines Gesellschafters nicht aufgelöst, Art. 1870 C.civ., wohl aber die OHG-ähnliche société en nom collectif; Fortsetzungsklauseln sind zulässig, C.com. art. L221-15. Hier zeigt sich, dass die société en nom collectif, obwohl „juristische Person“, in starkem Maße personenbezogen ist. ${ }^{68}$

\section{Personengesellschaft, Körperschaft und Kapitalgesellschaft: fließende Übergänge}

Mit Rücksicht auf den Anwendungsbezug nehme ich die Kapitalgesellschaft als pars pro toto für die entsprechend verfasste Körperschaft. Vom deutschen Recht her gedacht ist der Unterschied zwischen personengesellschaftsrechtlicher und körperschaftlicher Organisation, trotz fließender Übergänge und Mischformen kraft Privat- bzw. Satzungsautonomie, als Grundmuster entscheidend für die Bestimmung der Regel- und der Ausnahmeverhältnisse. Beispiele dafür sind etwa Mehrheits- oder Einstimmigkeitsprinzip, Abstimmung nach Köpfen oder Beteiligung, Mindestorganisation, Selbst- oder Fremdorganschaft und Übertragbarkeit der Anteile.

Das europäische Gesellschaftsrecht ist für solche Einteilungen allerdings nicht hilfreich. Art. 54 AEUV sagt dazu nichts; Cartesio war eine rechtsfähige KG ungarischen Rechts, also außerhalb des Anwendungsbereichs der 1. und 2. gesellschaftsrechtlichen Richtlinie. Auch Art. 1 Abs. 2 f und g der Rom-I VO stellt nicht auf die Unterscheidung zwischen juristischer Person und sonstigen Gruppen ab. Die gesellschaftsrechtlichen Richtlinien zählen für ihren Anwendungsbereich die Gesellschaftsformen jeweils auf; das sind die Kapitalgesellschaften. Die Abstraktion wird vermieden; für die Personengesellschaften folgt daraus nichts. Die nationalen Gesellschaftsrechte gehen hier eigene Wege.

\section{a) Mebrheitsprinzip und Abstimmungsmodus}

Das Mehrheitsprinzip erscheint uns typisch für die körperschaftliche Verfassung. Nicht so in anderen Ländern. In der société civile gilt das Einstimmig-

68 Dondero, Droit des sociétés, 2010, Rdn. 613 ff: caractère fermé; GuYon, Droit des affaires, Tome 1. Droit commercial général et Sociétés, 12. Aufl., 2003, Rdn. 257, 274; Nitschke, aaO (Fn. 31), S. 197 f. 
keitsprinzip, C.civ. art. 1852, ebenso in der société en nom collectif, C.com. art. L221-6, aber nur soweit die Gesellschafter zuständig sind, hauptsächlich also bei Grundlagengeschäften, denn die SC und die SNC haben einen Geschäftsführer (gérant), der eine starke Stellung hat. ${ }^{69}$ Für die Vertragsgesellschaft société en participation wird ein gérant zumindest empfohlen.

Die amerikanische general partnership ist durch gleiche Verwaltungsrechte der Partner gekennzeichnet; die Vorstellung eines Geschäftsführers als Normalfall ist dem fremd; "centralized management" ist ein Merkmal der corporation, also der Kapitalgesellschaft. ${ }^{70}$ In der englischen general partnership, die ja Vertragsgesellschaft ist, gilt das Mehrheitsprinzip für gewöhnliche Angelegenheiten (ordinary matters), ${ }^{71}$ ebenso bei der limited partnership für die general partners. ${ }^{72}$ Dem entspricht der amerikanische Grundsatz der Mehrheitsentscheidung bei gewöhnlichen Geschäften nach $\$ 18$ h UPA, $\$ 401$ j RUPA.

In Personengesellschaften wird nach Köpfen abgestimmt, in Kapitalgesellschaften nach Beteiligung, könnte man meinen. Im englischen Recht gibt es aber auch Abstimmungen nach Köpfen, oder besser: Händen (show of hands $)^{73}$ in der company, also Kapitalgesellschaft, Sec. $282 \mathrm{ff}$ Companies Act; und bei der Kapitalgesellschaft kann ein einstimmiger Beschluss sämtlicher Aktionäre in die Kompetenz des board eingreifen. ${ }^{74}$

\section{b) Mindestorganisation, Selbst- oder Fremdorganschaft}

Bei den Kapitalgesellschaften kennen wir eine körperschaftliche Mindeststruktur. Organe sind die Mitglieder, meist verfasst in der Mitgliederversammlung und ein Geschäftsführungs- und Vertretungsorgan. Die Personengesellschaften können weitgehend auf eine solche Binnenstruktur verzichten; Geschäftsführung und Vertretung stehen den Gesellschaftern zu, differenziert nach Gesellschaftsform und -vertrag. Hier soll es jedoch bei den gesetzlichen Grundmodellen bleiben.

Dabei fällt auf, dass die französischen registrierten Gesellschaften einen Geschäftsführer (gérant) haben müssen, der nicht Gesellschafter zu sein braucht; Fremdorganschaft ist zulässig, der gérant kann auch eine juristische Person sein. Bei der SNC, sind, wie bei der OHG, alle Gesellschafter zum Geschäfts-

69 Dondero, aaO (Fn. 68), Rdn. 595.

70 M. A. Eisenberg, aaO (Fn. 42), S. 106; O’Kelley/Thompson, aaO (Fn. 60), S. 135 f.

71 Sec. 24 (8) Partnership Act 1890; vgl. ferner Lindley \& Banks, aaO (Fn. 19), 15-05.

72 Sec. 6 (5)(a) Limited Partnerships Act 1907; vgl. ferner Blackett/Haren, aaO (Fn. 50), 24.9; Lindley \& BANKs, aaO (Fn. 19) 31-02.

73 Sec. 282 (3), Sec. 283 (4), Sec. 284 (2), Sec. 285 (1) Companies Act 2006.

74 Davies/Worthington, Principles of Modern Company Law, 9. Aufl., 2012, Rdn. 14-15 f m.N. aus der Rspr. 
führer berufen, vorbehaltlich abweichender Regelung im Gesellschaftsvertrag, C.com. art. L.221-3. Der Geschäftsführer der société civile wird nach dem Gesellschaftsvertrag, sonst mit einfacher Mehrheit der Gesellschafter gewählt und abberufen, C.civ. art. 1846. Hat die Gesellschaft keinen Geschäftsführer, kann gerichtlich ein Notgeschäftsführer bestellt werden; dauert der irreguläre Zustand über ein Jahr an, wird die Gesellschaft aufgelöst, C.civ. art. 1847. Die Vorstellung, dass die Geschäftsführungsbefugnis an die Gesellschafter zurückfällt, gibt es hier also nicht.

Im Gegensatz dazu fällt bei der englischen company, also der Grundform der Kapitalgesellschaft, die Geschäftsführungs- und auch die Vertretungskompetenz auf die Gesellschafter zurück, wenn sie keinen director oder board of directors hat oder diese wegen Interessenkonflikts nicht handlungsfähig sind (default power). ${ }^{75}$ Insoweit sind die französische société civile und société en nom collectif "körperschaftlicher" als die englische company. Die englische partnership, ebenso wie die amerikanische, geht von einer gemeinsamen Geschäftsführung der partner aus. Die partner sind agents der übrigen partner (England) ${ }^{76}$ bzw. der partnership ${ }^{77}$ (RUPA). Die LLP, juristische Person und stark der company angenähert, folgt im Innenverhältnis dem Personengesellschaftsrecht, also nicht der körperschaftlichen Verfassung. ${ }^{78}$

\section{c) Übertragbarkeit der Anteile}

Die Frage nach der Übertragbarkeit der Anteile wurde bereits oben (III.2.) im Zusammenhang mit der rechtlichen Verselbständigung angesprochen. Das Merkmal „unabhängig vom Mitgliederbestand“ trifft weder auf die französische société noch auf die RUPA-partnership zu. Auch diese rechtlich verselbständigten Gesellschaften folgen insoweit dem Muster der Personengesellschaft und nicht der Körperschaft und juristischen Person im Sinne des deutschen Rechts.

\section{Bedeutung der Registrierung}

Für die Gesellschaft bürgerlichen Rechts gibt es kein Register, was zu einigen praktischen Unzuträglichkeiten führt, aber auch der Mühe enthebt, sich über

75 Davies/Worthington, aaO (Fn. 74), 14-11 m.N. zur Rspr.

76 Sec. 5 Partnership Act 1890; Partnership Law, Gemeinschaftsbericht 2003, aaO (Fn. 38), S. 8, 57; vertiefend Lindley \& Banks, aaO (Fn. 19) 2-02 f, 2-13, 12-05.

77 So auch im schottischen Recht, siehe Partnership Law, Gemeinschaftsbericht 2003, aaO (Fn. 38), S. 57.

78 Sec. 5(1) (b), 15 (c) LLP Act; Blackett/Haren, aaO (Fn. 50) 25.1, 25.4; Just, Englisches Gesellschaftsrecht, 2008, S. 11. 
die rechtliche Bedeutung der Registereintragung den Kopf zu zerbrechen. Für die OHG ist die Eintragung deklaratorisch, wenn ein Handelsgewerbe betrieben wird, in den anderen Fällen konstitutiv, aber lediglich was den Charakter als OHG angeht, nicht hinsichtlich der Existenz der Gesellschaft. Entsprechendes gilt für die Partnerschaftsgesellschaft. Die Eintragungswirkungen sind über die Umsetzung der 1. gesellschaftsrechtlichen Richtlinie hinausgehend im HGB geregelt. Darauf wird im PartGG verwiesen. Das schließt die Publizität der Vertretungsverhältnisse ein.

\section{Erwerb der Rechtsfähigkeit}

Die société en participation wird nach dem Willen der Gesellschafter nicht eingetragen; personnes morales sind nur die eingetragenen Gesellschaften. Der Vertrag einer société civile oder en nom collectif bedarf der Schriftform zu Beweiszwecken. Nach der Rechtsprechung Ende des 19. Jahrhunderts, die die Vorbehalte gegen rechtliche Verselbständigung - Mediatisierung - seit der französischen Revolution aufgab, genügte der Vertragsschluss zur Schaffung der personne juridique. Mit der Reform des Handelsgesellschaftsrechts 1966 wurde diese Vertragstheorie aufgegeben, die Eintragung ist nunmehr konstitutiv, C.com. art. L-210-6. Seit 1978 gilt das auch für die société civile, C.civ. art. $1842 .{ }^{79}$ Damit entspricht die Entstehung der personne morale dem Prinzip der Normativbestimmungen.

Die partnership - englisch oder amerikanisch - verlangt keine Registrierung. Nach RUPA ist immerhin eine freiwillige Registrierung (filing of statements bei der zuständigen einzelstaatlichen Behörde) vorgesehen, $\mathbb{} 303 .^{80}$ Die Entstehung der „entity“ folgt also der Vertragstheorie. Um den Status einer amerikanischen LLP zu erreichen, muss die Gesellschaft sich als solche beim zuständigen einzelstaatlichen Register eintragen lassen, sie muss eine entsprechende Bezeichnung führen, $\mathbb{S} \mathbb{S} 1001 \mathrm{f}$ RUPA.

Die englische limited partnership, die keine rechtlich verselbständigte Einheit ist, bedarf der Registrierung; ohne Registrierung handelt es sich um eine general partnership, Sec. 5 LP Act 1907. Daraus folgt, dass der an sich formfreie Gesellschaftsvertrag doch schriftlich niedergelegt werden muss. ${ }^{81}$ Die amerikanische limited partnership entsteht nach $\$ 201$ ULPA $^{82}$ mit der Registrie-

79 Zur Entwicklung Ferid/Sonnenberger, aaO (Fn. 34), Rdn. 2L17ff.

80 Die freiwillige Registrierung bestimmter Personengesellschaften schlägt K. Sснмidt, ZHR 177 (2013), 712, 728 f, für das deutsche Recht vor.

81 Sec. 8 Limited Partnership Act 1907; Lindley \& BANKs, aaO (Fn. 19), $29-16$.

82 Uniform Limited Partnership Act 2001 (http://www.uniformlaws.org/shared/docs/limited\%20partnership/ulpa_final_2001rev.pdf), in einer beträchtlichen Zahl von Einzelstaaten umgesetzt, u. a. in Kalifornien, nicht aber in New York; im Kommentar zu \201 
rung (oder zu einem späteren, in den Dokumenten angegebenen Datum). Darüber wird ein „certificate of existence“ ausgestellt, $\$ 209$ ULPA. Das verwundert nicht, da hier Publizität und Einhaltung von rechtlichen Voraussetzungen der Haftungsbeschränkung betroffen sind.

\section{Vorgesellschaft}

Mindestens die konstitutive Registrierung führt zur Frage nach der Vorgesellschaft. Sogar die englische partnership, die nicht rechtlich verselbständigt ist, wirft Fragen im Gründungsstadium auf, denn sie verlangt ja eine aktive wirtschaftliche Tätigkeit. ${ }^{83} \mathrm{Im}$ deutschen Recht genügt das Handelsgewerbe, eine Personengesellschaft zur OHG zu machen. Bei konstitutiver Eintragung gibt es zuvor eine Gesellschaft bürgerlichen Rechts. Der Frage, ob es zu dieser - in der rechtsfähigen Variante - eine Vorgesellschaft gibt, will ich nicht nachgehen. ${ }^{84}$ Bei der vorliegend vernachlässigten $K G$ zeigt ein Blick auf $\$ 176$ HGB, dass es keiner Vorgesellschaft bedarf, denn die KG ist auch vor Eintragung KG; die Haftungsbeschränkung hängt von der Kenntnis des Vertragspartners ab. Das Muster passt für die Gesellschaften, die nach der Vertragstheorie zustande kommen. Eine (noch) nicht registrierte englische limited partnership gilt als general partnership ohne Haftungsbeschränkung, Sec. 5 LP Act 1907. Die britische LLP kann vor der Eintragung durch Vereinbarungen der Partner untereinander gebunden werden, Sec. 5 (2) LLP Act 2000, nicht aber gegenüber Dritten. ${ }^{85}$ Handeln Gesellschafter vor der Eintragung für die Gesellschaft, sind sie persönlich berechtigt und verpflichtet und haften unbeschränkt. ${ }^{86}$ Ein Übergang der Verbindlichkeiten auf die Gesellschaft ist nicht vorgesehen, die Übernahme ist aber möglich. ${ }^{87}$

Der Gründungsprozess in Frankreich ist zweistufig; der Eintragung (immatriculation) geht eine Vorabveröffentlichung voraus; elektronische Verfahren bewirken aber eine beträchtliche Beschleunigung. ${ }^{88}$ Der C.civ. kennt eine

heißt es: „A limited partnership is a creature of statute“. Zur Entwicklung RöDER, 78 RabelsZ 109, $118 \mathrm{ff}$.

83 Lindley \& Banks, aaO (Fn. 19) 2-05.

84 Gegen einen „Import“ des Vorgesellschaftsproblems in die Personengesellschaft K. SCHMIDT, ZHR 177 (2013), 712, $729 \mathrm{f}$.

85 Blackett/Haren, aaO (Fn. 50), 25.12.

86 Blackett/Haren, aaO (Fn. 50), 25.12 m. w. N.; Sec. 51 Companies Act 2006 ist durch die LLP Regulations 2009, SI 2009/1804, reg 7 in modifizierter Form auf die LLP anwendbar: "A contract that purports to be made or on behalf of an LLP at a time when the LLP has not been formed has effect, subject to any agreement to the contrary, as one made with the person purporting to act for the LLP or as agent for it, and he is liable on the contract accordingly."

87 Blackett/Haren, aaO (Fn. 50), 25.12.

88 Cozian/Viandier/Deboissy, aaO (Fn. 15), Rdn. 178 ff; zum Registerverfahren Son- 
Handelndenhaftung, die bei der société en nom collectif zur gesamtschuldnerischen Haftung, C.com. art. L210-6, bei der société civile zur Einzelhaftung, C.civ. art. 1843, führt. Auch Dritte können Handelnde sein, etwa der gérant. Die Gesellschaft kann die Engagements aber in verschiedenen rechtstechnischen Varianten übernehmen, u. a. in Form einer gewissen Automatisierung der Übernahme. ${ }^{89}$ Auf das Verhältnis der Gesellschafter untereinander findet bis zur Eintragung Schuldvertragsrecht Anwendung, C.civ. art. 1842. Bei Verzögerung der Eintragung wird die Gesellschaft als société de fait behandelt, ${ }^{90}$ auf die die Vorschriften über die société en participation, also die einfache, nicht rechtsfähige Vertragsgesellschaft, Anwendung finden, C.civ. art. 1873. Das schützt freilich nicht vor persönlicher Haftung (s. u. unter VI.).

\section{Publizität}

Die Publizitätsfolgen der Registrierung variieren nach dem jeweiligen handelsrechtlichen oder allgemeinen Regime. Insbesondere die Vertretungsverhältnisse können nicht allein durch Registerpublizität erfasst werden, zumal die 1. gesellschaftsrechtliche Richtlinie ${ }^{91}$ auf Personengesellschaften keine Anwendung findet. Ferner ist zwischen freiwilliger und obligatorischer Registrierung zu unterscheiden. Auf diese Fragen ist nachfolgend vor allem unter materiellrechtlichen Gesichtspunkten einzugehen.

\section{Vertretung und das ultra-vires-Problem}

Ein Punkt darf als erledigt angesehen werden: Bei der Vertragsgesellschaft (aggregate theory, société en participation) können nur die übrigen Gesellschafter vertreten werden, während bei rechtlicher Verselbständigung die Gesellschaft selbst vertreten wird. Fraglich bleiben die Reichweite der Verselbständigung der Gesellschaft und, eng damit verbunden, die Vertretungsmacht im Außenverhältnis und deren Nachweis. Im deutschen Handelsrecht haben wir grundsätzlich unbeschränkte rechtliche Verselbständigung; Rechtsfähigkeit wird, auch bei der GbR, umfassend verstanden, die Rede von der „Teilrechtsfähigkeit" stößt auf Kritik. ${ }^{92}$ Korrespondierend dazu ist die handels-

nenberger/Dammann, Französisches Handels- und Wirtschaftsrecht, 3. Aufl., 2008, Rdn. II $69 \mathrm{ff}$; III $116 \mathrm{ff}$.

89 Cozian/Viandier/Deboissy, aaO (Fn. 15), Rdn. 195.

90 Zur société de fait Nitschke, aaO (Fn. 31), S. 151 ff; zur société de fait als Vorbild für die fehlerhafte Gesellschaft OechsLer, NJW 2008, 2471, 2472 ff.

91 Publizitätsrichtlinie, kodifizierte Fassung 2009/101/EU v. 16. 9. 2009, ABl.EU L 258.

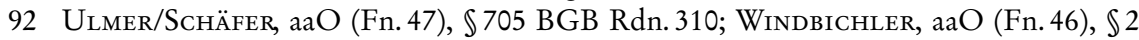
Rdn. 6.; zur Problematik des Umfangs der Rechtsfähigkeit allgemein Wiedemann, Gesellschaftsrecht, Band 1, 1980, \$4 II 2. 
rechtliche Vertretungsmacht flächendeckend unbeschränkt und unbeschränk-

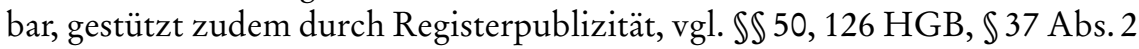
GmbHG, $\$ 82 \mathrm{AktG}$. Das ist ebenso einfach wie radikal. Die Schwelle für den Missbrauchseinwand ist hoch (Kollusion). Selbst Art. 10 Abs. 1 Satz 2 der 1. gesellschaftsrechtlichen Richtlinie ist demgegenüber zurückhaltender und lässt in engen Grenzen die Berufung auf die Grenzen des Unternehmensgegenstands zu.

Der Geschäftsführer der französischen eingetragenen Gesellschaften hat Vertretungsmacht im Rahmen des Unternehmensgegenstandes, C.civ. art. 1849, C.com. art. L221-5. Die französische Idee der personne morale geht von der Rechtsfähigkeit im Rahmen des objet social aus, dem Prinzip der spécialité, was sich in der Vertretungsregelung widerspiegelt. Unbeschränkt haftende Gesellschafter sollen nur im Rahmen des Unternehmensgegenstandes dem Risiko ausgesetzt werden; der Dritte muss sich bei Überschreitung an den Geschäftsführer halten. ${ }^{93}$ Auf gesellschaftsvertragliche Beschränkungen kann sich die Gesellschaft nicht berufen, wohl aber der Dritte. ${ }^{94}$ Besteht die Überschreitung der Vertretungsmacht in der Verletzung des Gesellschaftsinteresses, gelten die Grundsätze des détournement de pouvoir, die zur Nichtigkeit führen; gutgläubige Dritte sind jedoch geschützt. ${ }^{95}$ In Zweifelsfällen helfen die Grundsätze der Anscheinsvollmacht (mandat apparent). ${ }^{96}$

Die englische partnership kann die Vertretungsmacht der partner (für die übrigen partners) näher bestimmen; jedenfalls für gewöhnliche Geschäfte gilt die sog. „implied“ oder „apparent authority“, Sec. 5 Partnership Act 1890. ${ }^{97}$ Das ist die traditionelle Lösung, die die ultra vires-Problematik, die ursprünglich eine Frage der Reichweite der Rechtsfähigkeit ist, als Vertretungsproblem aufgreift. ${ }^{98}$ Partner einer schottischen partnership vertreten die Gesellschaft selbst, ${ }^{99}$ die Schranken sind aber dieselben. Auch die LLP (juristische Person, der $\mathrm{GmbH}$ angenähert) kennt keine unbeschränkte und unbeschränkbare Vertretungsmacht der Partner; die Vertretungsmacht kann im Gesellschaftsver-

93 Cozian/Viandier/Deboissy, aaO (Fn. 15), Rdn. 264, 277, $1138 \mathrm{f}$ (für die SNC), 1182 (für die SC); Dondero, aaO (Fn.68), Rdn.571, 583; C. Frey, aaO (Fn.17), S. 230; Guyon, aaO (Fn. 68), Rdn. 266; Nitschke, aaO (Fn. 31), S. 122 ff.

94 Cozian/Viandier/Deboissy, aaO (Fn. 15), Rdn. 265.

95 Cozian/Viandier/Deboissy, aaO (Fn. 15), Rdn. 266, 369.

96 C. Frey, aaO (Fn. 17), S. 193 ff.

97 Partnership Law, Gemeinschaftsbericht 2003, aaO (Fn. 38), S. 57 f; Lindley \& Banks, aaO (Fn. 19), 12-11 und 12-10 mit Zusammenfassung zur Wirkung von Sec. 5 Partnership Act 1890.

98 Zimmer, Internationales Gesellschaftsrecht, 1996, S. 241 ff; zum französischen Recht C. Frey, aaO (Fn. 17), S. 221 ff, 233 ff; der Unterschied zwischen begrenzter Rechtsfähigkeit und begrenzter Vertretungsmacht zeigt sich bei der Genehmigungsfähigkeit von ultra-vires-Geschäften.

99 Vgl. Partnership Law, Gemeinschaftsbericht 2003, aaO (Fn. 38), S. 57. 
trag geregelt, Dritten aber nur bei Kenntnis entgegengehalten werden. ${ }^{100} \mathrm{Da}-$ rin liegt eine Abweichung vom generellen agency law und auch vom partnership law; die Wendung "for the purpose of the business of the partnership" fehlt in Sec. 6 (1) LLP Act 2000.

Nach $\ 301$ Abs. 1 RUPA, in Abweichung vom UPA, genügt es, dass der Partner im Rahmen des Unternehmensgegenstands handelt: „for apparently carrying on in the ordinary course the partnership business or business of the kind carried on by the partnership“. Die partnership (legal entity, aber nicht notwendig registriert) kann andere Beschränkungen der Vertretungsmacht Dritten nur entgegenhalten, wenn diese positive Kenntnis oder eine Mitteilung erhalten hatten (received notification). Über den Unternehmensgegenstand hinausgehende Geschäfte binden die Gesellschaft nur, wenn die übrigen Gesellschafter zustimmen, $\$ 301$ Abs. 2 RUPA. Die freiwillige Registrierung kann die Vertretungsverhältnisse bindend klarstellen, $\$ 303$ RUPA.

Wir finden hier jeweils die Anknüpfung an die allgemeinen Regeln des Vertretungsrechts, auch zur erleichterten Vertretung im Geschäftsverkehr, in den jeweiligen Rechtsordnungen. Die englische und schottische Law Commission ist in diesem Punkt besonders zurückhaltend und empfiehlt nur vorsichtig Änderungen im Gesellschaftsrecht, das nicht sedes materiae sei, und verweist im Übrigen auf das weiterentwickelte agency law. ${ }^{101}$ Auch die amerikanische Rechtsprechung und Literatur verweist auf allgemeines agency law. ${ }^{102}$

\section{Haftungsverbältnisse}

Soweit die Gesellschaft ein Gesellschaftsvermögen hat, haftet sie mit diesem. Uns interessiert die Haftung der Gesellschafter. Dazu habe ich vorab schon Einiges gesagt, denn die Haftung hängt ganz wesentlich von den Verpflichtungs- und Vertretungsverhältnissen ab. Deshalb hier nur einige Ergänzungen.

Die société civile und die société en nom collectif habe ich bisher quasi in einem Atemzug behandelt; beide Formen haben viel gemeinsam. Bei der Haftung dagegen gibt es spürbare Unterschiede. In beiden Gesellschaften haften die Gesellschafter unbeschränkt, aber subsidiär. Die Gesellschafter der société civile haften erst, wenn die Gesellschaft zuvor vergeblich in Anspruch genommen worden ist, dann pro rata entsprechend ihrer Beteiligung, C.civ. artt. 1857, 1859. Beim Gesellschafter der OHG-ähnlichen société en nom collectif genügt es, dass die Gesellschaft in Verzug gesetzt wurde; dann haften die Gesellschafter als Gesamtschuldner, C.com. art. L221-1. Die Gesellschafter

100 Sec. 6 (2) LlP Act 2000. Vgl. Blackett/Haren, aaO (Fn. 50), 25.62-65.

101 Vgl. Partnership Law, Gemeinschaftsbericht 2003, aaO (Fn. 38), S. 61 f (Nr. 6.19), 304 (Nr. 20.13).

102 Z.B. O’Kelley/Thompson, aaO (Fn. 60), S. 72 m. w. N. 
einer société en participation haften nach vertragsrechtlichen Grundsätzen, d. h. nur, wenn sie selbst kontrahiert haben oder in ihrem Namen gehandelt wurde. Treten die Gesellschafter Dritten gegenüber als solche auf, haften sie, je nach Art der Geschäfte, wie Gesellschafter einer SC oder SNC, C.civ. art. 1872-1. Besteht das Gesellschaftsvermögen in einer indivision, gelten aber zugleich die Haftungsregeln für die Verwaltung der Rechtsgemeinschaft. Der commanditaire hat, wie im deutschen Recht, seine Einlage zu erbringen und haftet dann nicht mehr nach außen. Fehlt es an dieser Voraussetzung, kann er von den Gläubigern der Gesellschaft in Anspruch genommen werden. ${ }^{103}$

In der englischen partnership sind die Gesellschafter gemeinsam verpflichtet (jointly, je nach Anspruchgrundlage, and severally). ${ }^{104}$ Die Gesellschafter können aber unter dem Namen der partnership verklagt werden und klagen. ${ }^{105}$ Die Frage der Subsidiarität stellt sich hier nicht mangels rechtlicher Verselbständigung der Gesellschaft, wohl aber in Schottland; dort ist die Gesellschafterhaftung subsidiär.

Nach $\$ 306$ a RUPA haften die partner gesamtschuldnerisch (jointly and severally) für alle Verbindlichkeiten der Gesellschaft. Das ist eine Veränderung gegenüber $\$ 5$ UPA, wonach die Haftung je nach Anspruchsgrundlage gesamtschuldnerisch bei unerlaubter Handlung (tort, die Verantwortlichkeit der nicht rechtsfähigen - partnership stand in $\$ 13$ ) oder nur gemeinsam bei Ansprüchen aus Vertrag (jointly) war. Diese Differenzierung findet sich auch noch im englischen Recht, die Law Commission empfahl den Übergang allgemein zur gesamtschuldnerischen Haftung. ${ }^{106}$ Die Haftung ist bei den amerikanischen partnerships subsidiär, Gesellschaft und Gesellschafter müssen jeweils für sich verklagt werden, $\$ 307$ c RUPA. Gleichwohl können die Klagen gleichzeitig erhoben werden; die Subsidiarität wird über die Vollstreckungsreihenfolge gewährleistet, $\$ 307 \mathrm{~d}$ RUPA. Limited partners einer limited partnership nach ULPA haften nach außen überhaupt nicht, $\mathbb{\$} 303$, auch wenn sie im Innenverhältnis Managementaufgaben wahrnehmen. ${ }^{107}$

Zusammenfassend lassen sich immerhin aus deutscher Sicht erstaunliche Gemeinsamkeiten feststellen, nämlich die Subsidiarität der persönlichen Haftung und die Bindung an den Unternehmensgegenstand.

103 Vgl. C.civ. art. 1166.

104 Sec. 9 und 12 Partnership Act 1890; vgl. Lindley \& Banks, aaO (Fn. 19), 13-01 ff.

105 Lord Mackay of Clashfern, aaO (Fn. 53), 74.

106 Partnership Law, Gemeinschaftsbericht 2003, aaO (Fn. 38), S. 304 f (Nr. 20.15).

107 Darin liegt eine wesentliche Veränderung, eingeführt durch den RUPA 1997, gegenüber dem früheren Recht, das zur persönlichen Haftung bei Einmischung in die Geschäftsführung führte; $\$ 7$ ULPA 1016. 


\section{Sonderformen für bestimmte Zwecke}

Wie schon eingangs gesagt gibt es Sonderformen für bestimmte Zwecke. Das ist ein Biotop voller Diversität für sich. Einige Aspekte lassen sich allgemein festhalten. Bei Gesellschaften für Träger freier Berufe bedarf es stets einer Harmonisierung mit dem Berufsrecht, die auf unterschiedliche Weise bewerkstelligt werden kann. Das können Einschränkungen sein - wer darf Gesellschafter sein? - oder Instrumente wie im neuen $\$ 8$ PartGG: Pflichtversicherung als „Preis“ für Haftungsbeschränkung. In Frankreich gibt es z.B. die société civile professionelle ${ }^{108}$ sowie eine Fülle weiterer Spezialformen. Die englische LLP, die nicht mehr als Personengesellschaft angesehen wird, ist speziell für Anwälte und Wirtschaftsprüfer geschaffen worden. 109

Mir sind zwei, aus deutscher Sicht merkwürdige, Varianten von Personengesellschaften aufgefallen. Der Code civil sieht vor, dass bei besonderer gesetzlicher Zulassung Personengesellschaften den Kapitalmarkt in Anspruch nehmen können, C.civ. art. 1841. Davon wird im Immobiliensektor kräftig Gebrauch gemacht. Es gibt eine Vielzahl von „sociétés civiles de placement immobilier faisant publiquement appel à l'epargne" (so heißt die spezialgesetzlich zugelassene Form; es gibt noch eine Reihe anderer). Als Geschäftsführer haben sie regelmäßig eine juristische Person. Als sociétés civiles bringen diese Gesellschaften die (subsidiäre) persönliche Haftung der Gesellschafter mit sich. Treibende Kraft ist, wie so oft, die steuerliche Transparenz bzw. Flexibilität; Frankreich kennt diesbezüglich Wahlrechte. Aber auch ein anderer Gesichtspunkt mag eine Rolle spielen: Die Form der Personengesellschaft mit einem Initial- und einem Maximalkapital erlaubt so etwas wie eine Stufengründung.

Der andere auffällige Sonderfall sind die MLPs, die Master Limited Partnerships. Das sind amerikanische limited partnerships in bestimmten Geschäftsbereichen, deren Anteile öffentlich gehandelt werden. ${ }^{110}$ Auch hier sind steuerrechtliche Erwägungen maßgebend. ${ }^{111}$ Die „unitholders" sind aber als limited partners dem Risiko ausgesetzt, dass im Krisenfall Gläubiger sämtliche Ausschüttungen zurückholen können für Forderungen, die vor der Ausschüttung entstanden sind, auch wenn der Gesellschafter inzwischen ausgeschieden ist. Limited partnerships, die nicht unter den steuerlichen Anwendungsbereich der MLP fallen, tauchen gleichwohl mit handelbaren, börsennotierten Antei-

108 Cozian/Viandier/Deboissy, aaO (Fn. 15), Rdn. $1249 \mathrm{ff}$.

109 Blackett/Haren, aaO (Fn. 50), 25.1, 25.20; vgl. auch Henssler/Mansel, NJW 2007, 1393, 1394: hybride Gesellschaftsform.

110 Indices werden z. B. von der Firma Alerian angeboten (www.alerian.com).

111 RILEY, http://www.investopedia.com/articles/basics/07/ml_partnerships.asp. 
len auf. ${ }^{112}$ Über die privatrechtliche und ökonomische Sinnhaftigkeit mag man streiten; das Terrain ist hier dem Kapitalmarktrecht überlassen.

\section{Zusammenfassung: Legende für die Landkarte}

Was ich bis jetzt vorgestellt habe, ist immer noch keine Landkarte, sondern eher die Beschreibung von schroffen Bergen und sanften Hügeln, manchmal auch Sümpfen. Man mag sich fragen, ob man nach diesem Befund die Rechtsvergleichung nicht doch als übermäßig mühsam und unfruchtbar sein lassen sollte. Das bestreite ich natürlich heftig.

Eine Frage ist: Was kann und soll das Personengesellschaftsrecht, ja das Gesellschaftsrecht insgesamt eigentlich leisten? Welche Differenzierungen brauchen wir hinsichtlich der rechtlichen Verselbständigung? Die ultra vires-Problematik beleuchtet einen Aspekt des Verständnisses der juristischen Person überhaupt: Folgt man einer Gleichstellungstheorie in dem Sinne, dass eine juristische Person dieselben Kompetenzen hat wie eine natürliche Person (für den beschränkten Bereich der Niederlassungsfreiheit tut das Art. 54 AEUV), oder belässt man es bei einer Rechtsschöpfung mit diversen Varianten, wiederum nicht absolut gesehen sondern als Grundmodell zur Bestimmung der Regel-/ Ausnahmeverhältnisse?

In puncto Vermögensträgerschaft spiegelt sich die unterschiedliche Bereitschaft in den verschiedenen Rechtsordnungen, getrennte Vermögensmassen zuzulassen. Deutschland ist vergleichsweise zurückhaltend, während sich Frankreich und die angelsächsischen Länder damit leichter tun. In Frankreich wurde 2007 die "fiducie" eingeführt - als mögliche Konkurrenz zur Gesellschaft? -, die sich natürlich nicht an den englischen trust anlehnt, sondern an den gemeinsamen mittelalterlichen Vorfahr „fiducia“, wenn der Kreuzritter gen Jerusalem zieht und sein Besitztum zu Hause verwalten lassen muss. ${ }^{113}$

Auch in den Formen und Rechtsordnungen, die Personengesellschaften kennen, die nicht selbst Vermögensträger sind, findet eine Separierung des „Gesellschaftsvermögens" vom Privatvermögen der Gesellschafter statt. Das kennen wir von der Gesamthand als Vermögenszuordnung, ${ }^{114}$ nicht nur bei

112 Bekannt ist z.B. The Blackstone Group L.P., deren unitholders fast keine Rechte haben, vgl. SEC Form S-1/A vom 1. Mai 2007, http://ir.blackstone.com/secfiling.cfm?filing $I D=1047469-07-3476$.

113 C.civ. art. $2011 \mathrm{ff}$; Cozian/Viandier/Deboissy, aaO (Fn. 15), Rdn. $71 \mathrm{ff}$.

114 Außerhalb des Begriffswandels zum „Subjekt“, vgl. Ulmer/Schäfer, aaO (Fn. 47), \705 BGB Rdn. 296 ff; die rechtlich verselbständigte und vermögenstragende Gesellschaft braucht eigentlich keine Gesamthand zur Vermögenszuordnung mehr; vgl. K. Schmidt, AcP 209 (2009), 181, $196 \mathrm{ff}$; zur Vermögenstrennung Wiedemann, aaO (Fn. 92), \4 I 2 b, $\$ 5$ I. 
Gesellschaften, sondern auch bei anderen Gesamthandsgemeinschaften. Selbst beim Bruchteilseigentum besteht eine Trennung vom sonstigen Vermögen der einzelnen Bruchteilsinhaber, indem es z. B. einer Koordination der Mehrzahl von Berechtigten bedarf und die Gemeinschaft erst aufgehoben werden muss, bevor ein Gläubiger den Anteil verwerten kann. Die gemeinsame Zuständigkeit begründet freilich noch keine Gesellschaft; die Übergänge zwischen (sachenrechtlichem) Halten und Verwalten und gemeinsamem Zweck sind fließend. Das zeigt sich besonders deutlich am Verhältnis von indivision und société en participation. Seit 1976 behandelt der Code civil die vereinbarte Verwaltung der indivision, einem Phänomen des Sachenrechts, im Schuldrecht. ${ }^{115}$ Die $\int \mathbb{S} 741 \mathrm{ff}$ BGB dagegen beschränken sich nicht auf die vereinbarte Verwaltung der Rechtsgemeinschaft. Auch das Prinzip der Vermögenstrennung kennt also Abstufungen. Die persönliche Haftung ist normal und entspricht der Verkehrsanschauung. Aber in ihrer Reichweite und Ausgestaltung ist sie bei der OHG deutlich strenger als in den hier betrachteten anderen Rechtsordnungen. Aus dem Vorlesungsbetrieb kann ich berichten, dass auch in Deutschland das laienhafte Bauchgefühl subsidiäre Haftung annimmt.

Die Unterscheidung von körperschaftlicher Verfassung und personenbezogener Organisation erscheint mir wichtiger als die Einordnung als „juristische Person". ${ }^{116}$ Hier zeigen sich erhebliche rechtskulturelle Unterschiede. In Frankreich ist sogar die einfache Personengesellschaft nach deutschem Verständnis quasi-körperschaftlich verfasst; der britische Companies Act lässt dagegen auch für Kapitalgesellschaften personenbezogene Ausgestaltungen $\mathrm{zu}$, ähnlich unserer flexiblen $\mathrm{GmbH}$.

Eine andere Frage des generellen Grundverständnisses ist, ob Gesellschafter einer rechtlich verselbständigten Gesellschaft Treuepflichten untereinander, ggf. welcher Art haben. Teilweise wird die Treuepflicht als Grundelement der Personengesellschaft angesehen. In manchen Rechtsordnungen gilt das nicht, z. B. für die limited partners einer amerikanischen limited partnership, $\$ 305$ ULPA, allerdings nur in Bezug auf "fiduciary duties"; "good faith and fair dealing" bei der Ausübung der Gesellschafterrechte sind davon nicht berührt. Das Treuepflicht-Thema habe ich weitgehend ausgespart, weil sich dahinter ganz erhebliche pfadabhängige Unterschiede verbergen, deren Behandlung den Rahmen dieses Beitrags sprengen würde. Man wird aber festhalten können, dass Standards wie Treuepflicht, fiduciary duty oder duty of

115 Ferid/Sonnenberger, aaO (Fn. 34), Rdn. 2L26, 2L501 behandeln die indivision weiter im Sachenrecht.

116 Zur Unterscheidung körperschaftliche Verfassung - juristische Person Hadding, aaO (Fn. 47), S. 154 f, 166 ff m. w. N.; mit Tendenz zur Vereinheitlichung Grunewald, Gesellschaftsrecht, 9. Aufl., 2014, $\$ 1 \mathrm{Rdn} .105 \mathrm{f}$, vor $\$ 8 \mathrm{Rdn} .1 \mathrm{f}$; die Unterscheidung betonend K. Schmidt, ZIP 2014, 493, 498 f; Wiedemann, aaO (Fn. 92), \$2 I 1. 
loyalty als Steuerung für Gesellschaften als symbiotische oder relationale Beziehungen geeigneter sind als starr formulierte Pflichten.

Hinsichtlich der Außenbeziehungen ist mir das deutsche Handelsrecht in seiner Klarheit und Strenge ${ }^{117}$ sympathisch (home-country bias?). Deutschland hat die 1. gesellschaftsrechtliche Richtlinie umfassend für das Handelsrecht, nicht nur für die Kapitalgesellschaften, umgesetzt. Anderswo blieb es bei den zurückhaltenderen Regeln für die Publizität und Vertretung von Personengesellschaften. Das ist ein Gesichtspunkt, der bei der weiteren Entwicklung des Rechts der GbR zu erwägen ist. Zwar zeigt auch die französische Gesetzgebung eine starke Anlehnung der société civile an die société en nom collectif. ${ }^{118}$ Letztere ist aber sowohl hinsichtlich der Vertretung wie der persönlichen Haftung nicht so strikt wie die OHG.

Der Bedarf an einer kleinen, unkomplizierten Gesellschaft, mit oder ohne Gesellschaftsvermögen, lässt sich nicht leugnen. ${ }^{119}$ Man sollte nicht die studentische Wohngemeinschaft oder die Kindergartenfahrgemeinschaft, die den Fehler macht, als solche aufzutreten und ein gemeinsames Mehrkind-Transportfahrrad anzuschaffen, in die Rechtsfähigkeit oder gar Registrierung treiben. Die französische Rechtsentwicklung zeigt das: Mit der Einführung der Eintragungspflicht der société civile wurde der Bedarf an einer flexiblen und einfachen Form des Zusammenwirkens, auch im Hinblick auf gemeinsames Vermögen, deutlich und schlug sich in der Reform der indivision und der société en participation nieder. ${ }^{120}$ Letztere zeigt sich auch geeignet für große, aber beschränkte Projekte. Bei unternehmenstragenden Gesellschaften sieht das anders aus. Die Strenge des deutschen HGB passt zum vergleichsweise engen Kaufmannsbegriff als Einstieg. Für Kleingewerbetreibende oder projektbezogene Gesellschaften muss das nicht so sein. Könnte man dafür nicht etwas anderes vorhalten als eine zur beinahe-OHG mutierten GbR?

Eine rechtspolitische Frage ist, ob man wenige flexible Grundformen gewissermaßen als Allzweckmöbel, ${ }^{121}$ eine Reihe von Spezialformen (freie Berufe, Landwirtschaft, Baugesellschaften) oder Fortbildung dispositiven Rechts für atypische Gestaltungen (z. B. die Publikumspersonengesellschaft) haben will. Frankreich neigt zu einer Fülle von Spezialformen, was vielfach als unübersichtlich empfunden wird. Großbritannien und die USA belassen es bei wenigen Grundformen, wobei auch hier eine Tendenz zu Ausdifferenzierungen

117 Zimmer, aaO (Fn. 98), S. 244: „am Ende der Skala“.

118 Vgl. die Synopse bei Nitschke, aaO (Fn. 31), S. 217 ff.

119 So schon seit langem K. Sснмidt, ZHR 177 (2013), 712, 715 ff m. w. N.

120 Nitschke, aaO (Fn. 31), S. 62 ff, 135 ff.

121 So tendenziell das britische Recht; für das deutsche Recht und als Reformvorschlag K. SснміDт, ZHR 177 (2013), 712, 722 ff, 730 ff; zu den ökonomischen Gründen für die Verselbständigung von Gesellschaften Kirchner, FS Raiser, 2005, S.181; vgl. auch Hansmann/Kraakman, FS Hopt, 2010, S. 746. 
festzustellen ist (z. B. LLP, LLC). ${ }^{122}$ Die Rechtstechnik, für die Verwendung von allgemein geregelten Gesellschaftsformen Sondervorschriften für bestimmte Verwendungen vorzusehen, enthält ebenfalls Verwirrungspotenzial. ${ }^{123}$

$\mathrm{Ob}$ es einen Bedarf für kapitalmarktgängige Personengesellschaften gibt, hängt stark mit dem Steuerrecht zusammen. Dahinter steht die grundsätzlichere Frage, welche Regelungen eigentlich solche des Gesellschaftsrechts, des Steuerrechts, des Vertragsrechts, des Kapitalmarktrechts oder sonstiger Regulierung sind. Die erwähnten französischen Baugesellschaften und amerikanischen master limited partnerships unterliegen jeweils der Kapitalmarktregulierung betreffend Zulassung, Prospekt etc. Der Steuergesetzgeber möge sich entscheiden, welche Voraussetzungen transparente Besteuerung rechtfertigen. Die Rechtsform sollte nicht maßgebend sein. In Deutschland wird das Steuerrecht als „unerwünschte Rechtsquelle des Gesellschaftsrechts“ bezeichnet, in Frankreich als "fâcheux" befunden. In Betracht kommen Wahlrechte und Kriterienkataloge. Das Thema der rechtsformneutralen Besteuerung liegt aber außerhalb des hier behandelten Kartenausschnitts.

Die Akzeptanz von Gesellschaftsformen verhält sich wie kommunizierende Röhren; sie hängt ganz wesentlich von den Alternativen ab. Schlecht ist, wenn eine Röhre fehlt. Eine „optimale“ Personengesellschaft im Sinne eines Harmonisierungsziels gibt es nicht. Das jeweilige rechtliche, wirtschaftliche, kulturelle und historisch gewachsene Umfeld bleibt prägend. 